\title{
Sensor Selection for Classification of Physical Activity in Long- Term Wearable Devices
}

This paper was downloaded from TechRxiv (https://www.techrxiv.org).

\section{LICENSE}

CC BY 4.0

SUBMISSION DATE / POSTED DATE

$10-01-2021 / 13-01-2021$

\section{CITATION}

Kirchner, Jens; Faghih-Naini, Samira; Bisgin, Pinar; Fischer, Georg (2021): Sensor Selection for Classification of Physical Activity in Long-Term Wearable Devices. TechRxiv. Preprint.

https://doi.org/10.36227/techrxiv.13551308.v1

$\mathrm{DOI}$

10.36227/techrxiv.13551308.v1 


\title{
Sensor Selection for Classification of Physical Activity in Long-Term Wearable Devices
}

\author{
Jens Kirchner, Samira Faghih-Naini, Pinar Bisgin, and Georg Fischer \\ Institute for Electronics Engineering \\ Friedrich-Alexander-University Erlangen-Nurnberg (FAU) \\ Erlangen, Germany \\ Email: jens.kirchner@fau.de
}

\begin{abstract}
Classification of physical activity based on the kNN algorithm is assessed with different combinations of sensors (from accelerometer, gyroscope, barometer) with respect to classification accuracy, power consumption and computation time. For that purpose, a wearable sensor platform is proposed and a study with 20 subjects is conducted. The combination of accelerometer and barometer is found to provide the best trade-off for the three criteria: It provides an F1 score of $94.96 \pm 1.73 \%$, while computation time and power consumption are reduced by $45 \%$ and $88 \%$, respectively, compared to the full sensor set.
\end{abstract}

\section{INTRODUCTION}

For monitoring of chronic diseases such as congestive heart failure $(\mathrm{CHF})$ or chronic obstructive pulmonary disease (COPD), physical activity can serve in a twofold way: as a marker of health status itself due to adaptation of a person's daily activities to its physical fitness; and as context information for other measurement quantities, e.g. for evaluation of heart rate or shortness of breath based on the current physical load (see, e.g., [1]-[3] and the NYHA classification of CHF [4]).

When such an activity classification module shall be developed for wearable devices in long-term use, accurate classification is one of the major requirement but not the only one: Energy efficiency for the sake of long wearing times depends on the power consumption of the sensors in use and on the amount of data that has to be transmitted to off-body data hubs. To keep the transmitted data low, on-board real-time classification is needed, hence, computation time is critical.

Therefore, we here compare the effect of sensor selection on the described requirements classification accuracy, energy consumption and computation time. Different combinations of sensors out of accelerometer, gyroscope and barometer are evaluated for typical activities from daily life: standing, walking, walking stairs and jogging. In future projects, the proposed activity classification module shall be combined with a mobile communication platform and additional sensors for ECG and heart/lung sounds as proposed by the authors [5]-[9].

\section{MEthods}

\section{A. Sensor Platform}

The wearable sensor platform is based on an Arduino Micro, which contains the ATmega32U4 microcontroller (Microchip Technology). Data is provided via I2C by the $10 \mathrm{DOF}$ sensor module GY86, which comprises the high-resolution barometric pressure sensor MS5611 (TE Connectivity; range $10 \mathrm{mbar}$ to 1200 mbar) as well as the MPU6050 (InvenSense Inc.) $3 \mathrm{D}$ accelerometer and $3 \mathrm{D}$ gyroscope, with ranges $\pm 4 \mathrm{~g}$ and $\pm 500^{\circ} \mathrm{s}^{-1}$ selected, respectively. The magnetic field sensor, which is also contained in the GY86, was not used. For data storage the microSD breakout board+ (Adafruit) was connected via SPI. Sensor sampling rate is limited by the writing speed on the SD card and amounted to $28 \mathrm{~s}^{-1}$. The entire platform is fixed at the test subject's upper body by use of a chest band (see Fig. 1).

The sensors are calibrated at the beginning of each measurement with the platform fixed at the test subject and the person taking an upright body position for $1 \mathrm{~s}$.

Energy is provided by a mobile power bank, which is fixed at the test subject's hip during measurement.

\section{B. Study}

The study was conducted with 20 healthy volunteers $(45 / 55 \%$ male/female) with age $(24 \pm 4)$ years (range 2138 years) and BMI $22 \pm 3$ (19-27).

The study protocol was divided into two parts, which provided the training and test data and had an approximate duration of $20 \mathrm{~min}$ and $30 \mathrm{~min}$, respectively. Both protocol stages comprised several phases of the activities standing in upright position, walking in the flat, walking upstairs, walking downstairs and jogging.

\section{Activity Classification}

For data analysis, Matlab ${ }^{\circledR}$ (MathWorks $^{\circledR}$ ), version 2017b, was used.

From both accelerometer and gyroscope, the three vector components and the absolute value of the $3 \mathrm{D}$ vector were obtained as measurement variables, as well as barometric pressure. This gives nine variables in total.

1) Data Preprocessing: Barometric pressure was smoothed by use of a Savitzky-Golay filter with width 121 samples and degree 1.

2) Feature Generation: For each measurement variable, the following quantities were derived in time space: (1) arithmetic mean [10], (2) root mean square value [11], (3-4) variance and standard deviation [10], (5) mean absolute deviation from mean [11], [12], (6-8) maximum, minimum and range [10], 


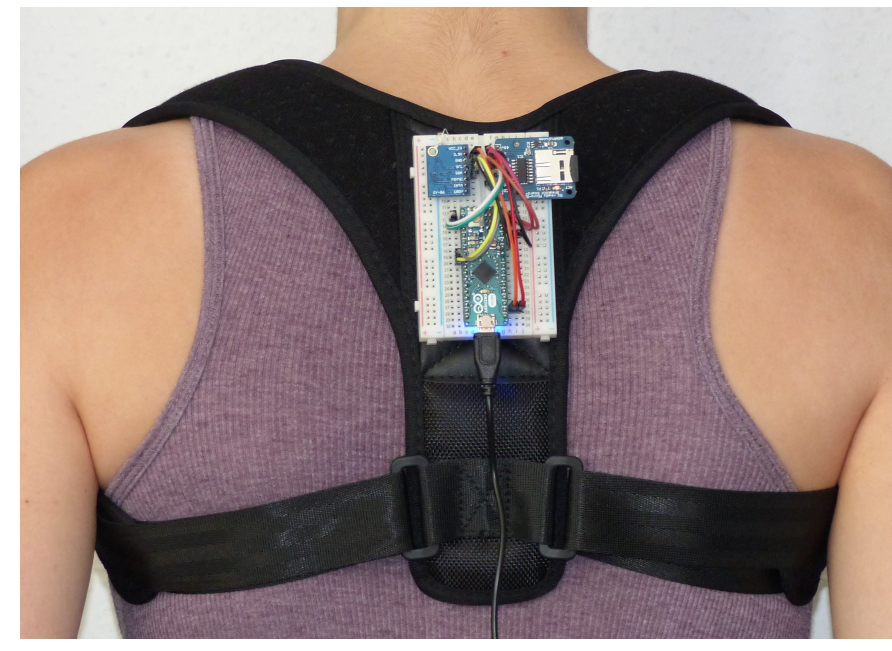

Fig. 1. Wearable sensor platform fixed at a test subject's upper body.

(9) slope, (10-11) numbers of zero and mean-value crossings [12], (12-13) number and mean amplitude of peaks with amplitude larger than $60 \%$ of the global maximum [13], (1415) skewness and curtosis [14], (16) inter-quartiles range [15], [16], (17) entropy [12], [17], (18) correlation coefficient [18], (19-20) signal magnitude area and signal vector magnitude [19], [20].

In frequency space, the following quantities were computed for each variable: (21-24) amplitude and frequency of the two largest maxima [10], [16], (25-26) energy and power [10], (27) ratio of maximum peak amplitude and total signal energy/power [10], (28) spectral centroid [12], (29) spectral entropy [21].

3) Normalization: Three different normalization strategies were investigated [22], [23]: (1) linear normalization on range $[-1,1]$; (2) linear normalization on range $[0,1]$; (3) standardization.

4) Feature Selection: Feature were selected by use of the ReliefF [24] and Rand Features [25]

5) Metrics: For the evaluating the distance to the next neighbors, the following metrics were evaluated [26]-[29]: (1) Euclidean distance, (2) City-block distance, (3) Chebyshev distance, (4) Correlation distance, (5) Spearman distance, (6) Cosine similarity.

6) Parameter Optimization: The free parameters were optimized with respect to the F1 score [30]

$$
\mathrm{F} 1=\frac{2 \cdot P \cdot S}{P+S},
$$

which combines the two common measures of classification accuracy, sensitivity $S$ and precision $P$

$$
S=\frac{\mathrm{TP}}{\mathrm{TP}+\mathrm{FN}} \quad P=\frac{\mathrm{TP}}{\mathrm{TP}+\mathrm{FP}}
$$

For a given activity $A$, TP denotes the number of $A$ windows that were correctly classified as $A$ ("true positives"), FP the number of non- $A$ windows that were falsely classified as $A$ ("false positives") and FN the number of $A$ windows that were falsely classified as non- $A$ ("false negatives").
7) Computation time: Computation time for each classification with a given parameter set was determined. In order to obtain a hardware-independent quantity, absolute computation time was normalized by the computation time for the full sensor set at optimal parameters (see section III with Tab. I).

\section{Power consumption}

The power consumed was derived from the data sheets of the senors for normal operating mode [31], [32].

\section{RESUlTS}

Optimization with all three sensors gave an optimal window width and overlap of 112 samples and $90 \%$, respectively. For the optimizations with a reduced sensor set, these parameters were kept fixed.

The results of the optimization of all remaining free parameters are summarized in Tab. I. With the optimal parameter set in the full sensor set a total F1 score of $(95.71 \pm 1.76) \%$ with sensitivity $(96.52 \pm 1.72) \%$ and precision $(94.93 \pm 2.00) \%$ is obtained. While the activities standing and jogging are well classified, distinguishing between the different ways of walking is more challenging and leads to lower classification performance.

With a reduced sensor set, classification performance worsens as expected. However, when the accelerometer together with either gyroscope or barometer is used, nearly the same accuracy is reached, with total F1 score, sensitivity and precision decreased by less than $1 \%$. With a single sensor, a total F1 score of $91 \%$ for accelerometer and gyroscope and of $62 \%$ for barometer is obtained.

Power consumption strongly depends on the sensor set used. With about $12 \mathrm{~mW}$, consumption of the gyroscope is by a factor 7.2 larger than the accelerometer and even four orders or magnitude larger than the barometer.

Computation time is reduced by up to $61 \%$ with accelerometer only and by $45 \%$ and $46 \%$ with accelerometer plus barometer and gyroscope plus barometer, respectively. A general trend, however, cannot be observed; instead, computation depends on the specific sensor set and its optimized parameters.

\section{CONCLUSION}

Sensor selection determines classification accuracy and computation time and has a large effect on power consumption: Leaving out the gyroscope reduces energy consumption by $88 \%$ and computation time by $45 \%$, while $\mathrm{F} 1$ score, sensitivity and precision are decreased by less than $1 \%$. Power consumption can further be decreased by using the barometric sensor only, yet at the expense of a considerable worsening of classification performance (F1 score $62.08 \%$ ). Hence, the sensor combination of accelerometer and barometer provides the best trade-off concerning classification accuracy, computation time and power consumption in our setup.

After fixing a specific sensor set, the three performance factors can be further tuned by adapting the free parameters of the classification algorithm. 


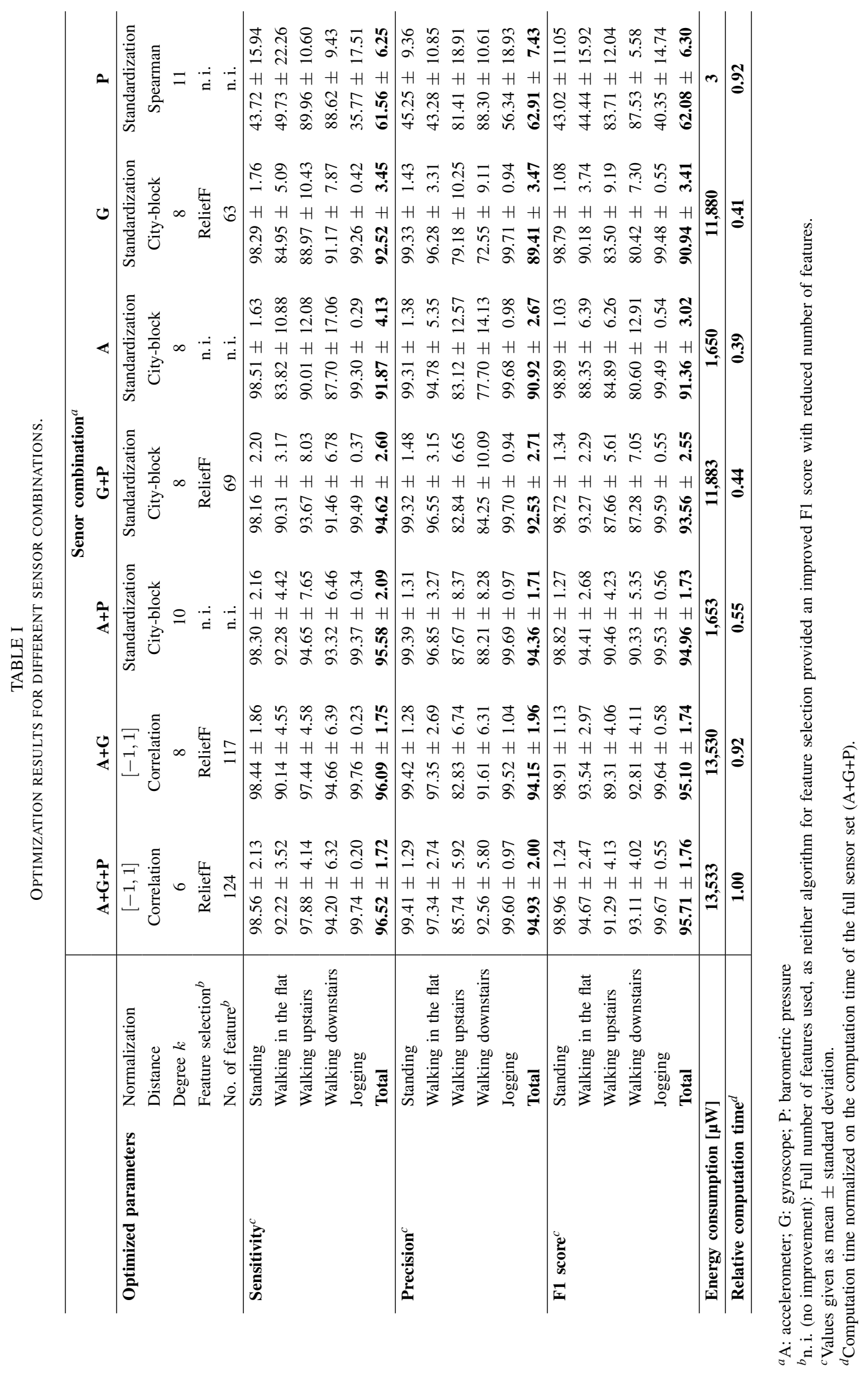




\section{REFERENCES}

[1] C. Bellos, A. Papadopoulos, R. Rosso, and D. I. Fotiadis, "Clinical validation of the chronious wearable system in patients with chronic disease," in 2013 35th Annual International Conference of the IEEE Engineering in Medicine and Biology Society (EMBC), 2013, pp. 70847087.

[2] G. Spina, P. Casale, P. S. Albert, J. Alison, J. Garcia-Aymerich, R. W. Costello, N. A. Hernandes, A. J. R. van Gestel, J. D. Leuppi, R. Mesquita, S. J. Singh, F. W. J. M. Smeenk, R. Tal-Singer, E. F. M. Wouters, M. A. Spruit, and A. C. den Brinker, "Identifying physical activity profiles in copd patients using topic models," IEEE Journal of Biomedical and Health Informatics, vol. 19, no. 5, pp. 1567-1576, 2015.

[3] D. Aranki, G. Kurillo, P. Yan, D. M. Liebovitz, and R. Bajcsy, "Realtime tele-monitoring of patients with chronic heart-failure using a smartphone: Lessons learned," IEEE Transactions on Affective Computing, vol. 7, no. 3, pp. 206-219, 2016.

[4] M. Dolgin and New York Heart Association. Criteria Committee, Nomenclature and criteria for diagnosis of diseases of the heart and great vessels, 9th ed. Boston, MA: Litte, Brown \& Co., 1994.

[5] R. Agethen, F. Lurz, A. Schwarzmeier, G. Fischer, R. Weigel, and D. Kissinger, "An online telemetering system for mobile health parameter monitoring and medical assistance," in Proc. 2011 5th Int. Conf. Sens. Technol. IEEE, 2011, pp. 470-473.

[6] A. Schwarzmeier, J. Besser, R. Weigel, G. Fischer, and D. Kissinger, "A compact back-plaster sensor node for dementia and alzheimer patient care," in Proc. 2014 IEEE Sens. Appl. Symp. IEEE, 2014, pp. 75-78.

[7] D. Kissinger, A. Schwarzmeier, F. Grimminger, J. Mena-Carrillo, W. Weber, G. Hofer, G. Fischer, and R. Weigel, "Wireless integrated sensor nodes for indoor monitoring and localization," in Proc. 2015 IEEE Top. Conf. Wirel. Sens. Sens. Netw. IEEE, 2015, pp. 7-10.

[8] J. Kirchner, N. Roth, A. Meyer, and G. Fischer, "ECG measurement by use of passive capacitively coupled electrodes," in Proc. 2016 IEEE Sensors, 2016, pp. 1-3.

[9] J. Kirchner, S. Souilem, and G. Fischer, "Wearable system for measurement of thoracic sounds with a microphone array," in 2017 IEEE SENSORS, 2017, pp. 1-3.

[10] A. Mannini, D. Trojaniello, A. Cereatti, and A. M. Sabatini, "A machine learning framework for gait classification using inertial sensors: Application to elderly, post-stroke and huntington's disease patients," Sensors, vol. 16 , no. $1,2016$.

[11] A. Mannini, S. S. Intille, M. Rosenberger, A. Sabatini, and W. Haskell, "Activity recognition using a single accelerometer placed at the wrist or ankle," Medicine and science in sports and exercise, vol. 45, 042013.

[12] A. Avci, S. Bosch, M. Marin-Perianu, R. Marin-Perianu, and P. Havinga, "Activity recognition using inertial sensing for healthcare, wellbeing and sports applications: A survey," 23th International Conference on Architecture of Computing Systems 2010, pp. 1-10, 2010.

[13] J. A. Ward, P. Lukowicz, and G. Tröster, "Gesture spotting using wrist worn microphone and 3-axis accelerometer," in Proceedings of the 2005 Joint Conference on Smart Objects and Ambient Intelligence: Innovative Context-aware Services: Usages and Technologies, ser. sOc-EUSAI '05. ACM, 2005, pp. 99-104.

[14] Y. C. Huang, C. W. Yi, W. C. Peng, H. C. Lin, and C. Y. Huang, "A study on multiple wearable sensors for activity recognition," in 2017 IEEE Conference on Dependable and Secure Computing, 2017, pp. 449-452.
[15] B. Luderer, V. Nollau, and K. Vetters, Mathematische Formeln für Wirtschaftswissenschaftler. Springer Gabler, 2015.

[16] O. D. Lara and M. A. Labrador, "A survey on human activity recognition using wearable sensors," IEEE Communications Surveys Tutorials, vol. 15, no. 3, pp. 1192-1209, 2013.

[17] C. E. Shannon, "A mathematical theory of communication," The Bell System Technical Journal, vol. 27, no. 3, pp. 379-423, 071948.

[18] N. Ravi, N. Dandekar, P. Mysore, and M. L. Littman, "Activity recognition from accelerometer data," in Proceedings of the 17th Conference on Innovative Applications of Artificial Intelligence Volume 3, ser. IAAI'05. AAAI Press, 2005, pp. 1541-1546. [Online]. Available: http://dl.acm.org/citation.cfm?id=1620092.1620107

[19] M. T. Redfield, J. C. Cagle, B. J. Hafner, and J. E. Sanders, "Classifying prosthetic use via accelerometry in persons with transtibial amputations," Journal of rehabilitation research and development, vol. 50, no. 9, pp. 1201-1212, 2013

[20] S. D. Bersch, D. Azzi, R. Khusainov, I. E. Achumba, and J. Ries, "Sensor data acquisition and processing parameters for human activity classification," Sensors, vol. 14, no. 3, pp. 4239-4270, 32014.

[21] A. Zhang, B. Yang, and L. Huang, "Feature extraction of eeg signals using power spectral entropy," in 2008 International Conference on BioMedical Engineering and Informatics, vol. 2, 05 2008, pp. 435-439.

[22] S. Aksoy and R. Haralick, "Feature normalization and likelihood-based similarity measures for image retrieval," Pattern Recognition Letters, vol. 22, 052000

[23] W. Tang and E. Sazonov, "Highly accurate recognition of human postures and activities through classification with rejection," IEEE journal of biomedical and health informatics, vol. 18, pp. 309-315, 012014.

[24] M. Robnik-Šikonja and I. Kononenko, "Theoretical and empirical analysis of relieff and rrelieff," Machine Learning, vol. 53, no. 1, pp. 23-69, Oct 2003. [Online]. Available: https://doi.org/10.1023/A: 1025667309714

[25] H. Liu and H. Motoda, Feature Selection for Knowledge Discovery and Data Mining. Kluwer Academic Publishers, 1998.

[26] K. Q. Weinberger, J. Blitzer, and L. K. Saul, "Distance metric learning for large margin nearest neighbor classification," in In NIPS. MIT Press, 2006

[27] L.-Y. Hu, M.-W. Huang, S.-W. Ke, and C.-F. Tsai, "The distance function effect on k-nearest neighbor classification for medical datasets," SpringerPlus, vol. 5, no. 1, p. 1304, Aug 2016. [Online]. Available: https://doi.org/10.1186/s40064-016-2941-7

[28] F. v. d. Heijden, Classification, parameter estimation, and state estimation : an engineering approach using MATLAB. John Wiley and Sons, Inc., 2017.

[29] M. R. Peterson, T. E. Doom, and M. L. Raymer, "Ga-facilitated knn classifier optimization with varying similarity measures," in 2005 IEEE Congress on Evolutionary Computation, vol. 3, Sept 2005, pp. 2514 2521 Vol. 3.

[30] A. Bulling, U. Blanke, and B. Schiele, "A tutorial on human activity recognition using body-worn inertial sensors," ACM Comput. Surv., vol. 46, no. 3, pp. 33:1-33:33, Jan. 2014. [Online]. Available: http://doi.acm.org/10.1145/2499621

[31] MPU-6000 and MPU-6050 Product Specification Revision 3.4, InvenSense, Inc.

[32] MS5611-01BA03 Barometric Pressure Sensor, with stainless steel cap. Measurement Specialties, Inc. 